\title{
Comparison of Short Tandem Repeat Loci D2S1338 and D18S51 in the Populations of the Republic of Srpska and the Autonomous Province of Vojvodina
}

\author{
Branka Samardžić, '̌̌ljkko Karan, ${ }^{1,2}$ Zoran Obradović, Dijana Došen ${ }^{1}$
}

\begin{abstract}
Introduction: Numerous human migrations between Bosnia and Herzegovina and Serbia's northern province of Vojvodina have led to the increased mixing of these population's genetic pool, leading to a reduction in genetic differences. The question is whether there are now genetic differences between the mentioned populations. Methods: Short Tandem Repeat (STR) loci D2S1338 and D18S51 were used in this paper to compare the diversity of alleles and genotypes between the populations of Vojvodina and one of the two Bosnia's entities, the Republic of Srpska (RS). Three hundred ninety unrelated persons, 140 persons from the RS and 260 from Vojvodina, were analysed. The PowerPlex ESX16 System commercial kit was used for profiling persons from the territory of the RS and the AmpF/STR Identifiler commercial kit for persons from Vojvodina. The Mann-Whitney U-test was used for statistical analysis.

Results: Data analysis concluded a significant deviation in the allele frequency of the D18S51 locus where $\mathrm{p}=0.021$. There was no statistically significant deviation in the D2S1338 locus allele frequency between these two populations. It was also found that there is a statistically significant deviation in the genotype frequencies of these two populations for the analysed genetic markers.

Conclusion: This study confirms the existence of a significant deviation of allelic frequency for the D18S51 gene locus and a significant deviation of both gene markers frequency of genotypes.
\end{abstract}

Key words: Human genome; Alleles; Microsatellite repeats; Population genetics.
(1) Institute of Forensic Medicine of Republic of Srpska, Banja Luka, the Republic of Srpska, Bosnia and Herzegovina.

(2) Faculty of Medicine, University of Banja Luka, Banja Luka, the Republic of Srpska, Bosnia and Herzegovina.

Correspondence: BRANKA SAMARDŽIĆ E: branka.zoric88@gmail.com

\section{ARTICLE INFO}

Received: 29 December 2020 Revision received: 21 March 2021 Accepted: 2 April 2021

\section{Introduction}

The DNA molecule consists of coding and non-coding regions. The non-coding parts are characterised by exceptional polymorphism. There are three main types of non-coding region polymorphisms:

- Minisatellites (Variable Number of Tandem repeats - VNTRs),

- Short Tandem Repeats (STRs),

- $\quad$ Single Nucleotide Polymorphisms (SNPs). ${ }^{1}$
STRs consist of short repetitive parts 2-10 base pairs long. These repetitions are widespread in the human genome and represent a rich source of highly polymorphic markers. Alleles of these loci differ in the number of repeats of the repetitive sequence located in the amplification region. ${ }^{2}$ STRs have such properties as abundant, codominant, highly polymorphic, and nearly selectively neutral which makes them very useful in applications such as the construction of genetic maps, gene location, genetic linkage analysis, identifi- 
cation of individuals, paternity testing, as well as disease diagnosis. STR analysis has also been employed in population genetics. They can be applied to reconstruct the history of migration and evolution of the species, as well as to assess biological diversity at various levels of biological organization. ${ }^{3}$

Numerous human migrations between Bosnia and Herzegovina and Serbia's northern province of Vojvodina have led to the increased mixing of these population's genetic pool, leading to a reduction in genetic differences. The question is whether there are now genetic differences between the mentioned populations.

This paper's aim was to determine and compare the frequencies of alleles and genotypes of STR loci D2S1338 and D18S51 in population of the Republic of Srpska (RS) and Vojvodina. Proposed hypothesis is that there are no genetic differences between two mentioned populations.

\section{Methods}

The diversity of alleles and genotypes of the two previously mentioned gene markers was analysed on a sample of 390 unrelated individuals. One hundred forty people were analysed from the territory of the RS, and 250 from the territory of Vojvodina.

Samples from the RS were collected in the Institute of Forensic Medicine of Republic of Srpska during of disputed paternity testing and analysis biological traces of human origin at the crime scene. DNA amplification was performed according to the PowerPlex ESX 16 Systems protocol in PCR ABI-Veriti, manufactured by Applied Biosystems, USA. Separation and detection of amplified fragments was performed by capillary electrophoresis on an instrument, ABI PRISM 310 Genetic Analyzer, manufactured by Applied Biosystems, USA. The analysis of the obtained fragments was performed using the software package GeneMapper ID v3.2.1. manufactured by Applied Biosystems, USA.

The Institute of Forensic Medicine in Novi Sad provided genotypes of 250 unrelated persons from Vojvodina. A commercial set AmpF/STR Identifiler, manufactured by Applied Biosystems, USA, was used for DNA amplification.

Statistical data processing was performed using the Mann-Whitney U-test.

\section{Results}

The results of the analysis of the diversity of 2 microsatellite loci D2S1338 and D18S51 in the human populations of the RS and Vojvodina are presented here. A total of 390 people were analysed.

The D2S1338 gene marker has 12 identified alleles in the analysed population of the RS and 14 alleles in the population of Vojvodina, allele 17 being the most common in both populations (Table 1). The D18S51 gene marker has 12 identified alleles in the analysed population of the RS and 16 alleles in the population of Vojvodina. Allele 14 has the highest frequency in the RS's population and allele 15 in the population of Vojvodina (Table 2).

Table 1: The allelic frequencies of D2S1338 locus \%

\begin{tabular}{|c|c|c|}
\hline Alleles & Republic of Srpska & Vojvodina \\
\hline 13 & 0.000 & 0.200 \\
\hline 15 & 0.357 & 0.400 \\
\hline 16 & 3.571 & 5.000 \\
\hline 17 & 18.214 & 21.600 \\
\hline 18 & 10.357 & 10.800 \\
\hline 19 & 11.786 & 9.200 \\
\hline 20 & 16.786 & 13.200 \\
\hline 21 & 3.929 & 3.000 \\
\hline 22 & 2.500 & 2.800 \\
\hline 23 & 15.000 & 10.800 \\
\hline 24 & 7.500 & 10.200 \\
\hline 25 & 8.214 & 11.200 \\
\hline 26 & 1.786 & 1.400 \\
\hline 27 & 0.000 & 0.200 \\
\hline
\end{tabular}

According to the Mann-Whitney U-test, the significance level is $p<0.05$. Data analysis concludes that there is no statistically significant deviation in the frequency of the alleles in the D2S1338 locus between these two populations. However, there is a statistically significant deviation in the allele frequency of the D18S51 locus where $p=0.021$. The allelic frequencies of these two loci in the populations of the RS and Vojvodina are shown in Table 3.

In the population of the RS, the presence of 49 different genotypes for both gene markers was determined. On the D2S1338 gene marker, the same frequency of 4 different genotypes was determined, while on the D18S51 gene marker, one genotype is singled out as the most common. In the sample from the Vojvodina territory, the presence of 57 
Table 2: The allelic frequencies of D18S51 locus \%

\begin{tabular}{|c|c|c|}
\hline Alleles & Republic of Srpska & Vojvodina \\
\hline 9 & 0.000 & 0.200 \\
\hline 10 & 1.786 & 1.000 \\
\hline 11 & 1.786 & 1.800 \\
\hline 12 & 10.357 & 13.000 \\
\hline 13 & 10.714 & 13.600 \\
\hline 14 & 20.357 & 16.000 \\
\hline 15 & 15.000 & 16.400 \\
\hline 16 & 13.571 & 15.000 \\
\hline 17 & 11.786 & 9.200 \\
\hline 18 & 5.000 & 8.000 \\
\hline 19 & 5.357 & 1.600 \\
\hline 20 & 2.143 & 2.200 \\
\hline 21 & 2.143 & 0.800 \\
\hline 22 & 0.000 & 0.800 \\
\hline 24 & 0.000 & 0.200 \\
\hline 25 & 0.000 & 0.200 \\
\hline
\end{tabular}

Table 3: Differences of allelic frequencies in loci according to Mann-Whitney U-test

\begin{tabular}{|c|c|c|c|c|c|}
\hline Loci & $\mathbf{U}$ & Z & p-value & Z-adjusted & p-value \\
\hline D2S1338 & 68362.50 & -0.542 & 0.588 & -0.547 & 0.584 \\
\hline D18S51 & 63068.50 & 2.296 & 0.022 & 2.317 & 0.021 \\
\hline
\end{tabular}

By variable Var1 Marked tests are significant at $p<0.05$ (red colour)

Table 4: Number of genotypes by loci

\begin{tabular}{|c|c|c|c|c|}
\hline \multirow[b]{2}{*}{ Loci } & \multicolumn{2}{|c|}{ Republic of Srpska } & \multicolumn{2}{|c|}{ Vojvodina } \\
\hline & A & B & A & B \\
\hline \multirow{4}{*}{ D2S1338 } & 49 & $20,23-8$ & 57 & $17,18-14$ \\
\hline & & $17,23-8$ & & \\
\hline & & $17,19-8$ & & \\
\hline & & $17,18-8$ & & \\
\hline \multirow{2}{*}{ D18S51 } & 49 & $14,15-11$ & 60 & $13,15-13$ \\
\hline & & & & $15,16-13$ \\
\hline
\end{tabular}

A. Number of genotypes in the population

$B$. The most common genotype-the number of repeats

Table 5: Summary of the difference of genotypic frequencies according to Mann-Whitney U-test

\begin{tabular}{cccccc}
\hline Loci & $\mathbf{U}$ & $\mathbf{Z}$ & $\mathbf{p}$-value & Z-adjusted & p-value \\
\hline D2S1338 & 14359.00 & -2.941 & 0.003 & -2.942 & 0.003 \\
\hline D18S51 & 14914.50 & -2.421 & 0.015 & -2.422 & 0.015 \\
\hline By variable Var1 Marked tests are significant at $p<0.05$ (red colour)
\end{tabular}

different genotypes for the D2S1338 gene marker and 60 genotypes for the D18S51 gene marker was determined. At the D2S1338 gene locus, one genotype is singled out as the most common, and at D18S51, we see the same frequency of 2 genotypes. A comparative overview of genotype's number in the analysed loci, in each population, as well as the most common combinations of alleles are shown in Table 4 . Table 5 shows the difference in genotype frequencies of these two populations for the analysed gene markers from which it can be seen that there is a significant deviation.

\section{Discussion}

Polymorphism of the D2S1338 and D18S51 gene markers was confirmed in both analysed populations (the RS and Vojvodina).

There are no earlier studies concerning similar research in RS, but there are several researches which include Bosnia and Herzegovina. In 2006, a survey was conducted for the territory of Bosnia and Herzegovina, which analysed 110 people. It was reported the presence of 11 alleles for D2S1338 marker with allele 17 being the most common allele. ${ }^{4}$ Later study covering 1000 persons from the same territory identified presence of 15 alleles in this STR marker, where allele 14 has the highest frequency. ${ }^{5}$ Locus D18S51 has shown bigger diversity in previous studies in Bosnia and Herzegovina. They found 15 allelic forms, with allele 16 being the one with the highest frequency. ${ }^{6}$

The sample of 100 unrelated, autochthonous healthy adult Serbians from Novi Sad (Vojvodina Province, Serbia and Montenegro) was profiled via AmpFLSTR Identifiler loci in 2004. Scientists have reported 12 alleles for STR marker D2S1338 (allele 17 had the highest frequency) and 14 for D18S51 marker (equally represented alleles 13 and 16$)^{7}$

Similar results to ours, concerning the number of allelic forms have been reported earlier from the countries in the region..$^{8,9}$

Comparison of allele diversity and genotypes of these gene markers was made using the Mann-Whitney U-test. A significant deviation in allele frequency was observed for the D18S51 gene marker, which was expected given that this 
locus showed high allelic diversity in both populations. Both analysed genetic markers showed a statistically significant deviation in the frequency of genotypes in the human populations of the RS and Vojvodina. It follows that there are specific genetic differences between the inhabitants of the RS and Vojvodina.

Since two gene markers with a high degree of genetic variability were analysed, caution is necessary in concluding the differences between these two populations. Many possible allelic forms characterise D2S1338 and D18S51 and a significant deviation could be expected.

\section{Conclusion}

In a sample of 390 unrelated persons from the territories of the Republic of Srpska and Vojvodina, allelic diversity was determined on both genetic markers. The autosomal marker D2S1338 has less allelic diversity than the range, while the allelic diversity is in the range for the marker D18S51. Based on this study, it can be concluded that a significant deviation of allelic frequency for the D18S51 gene locus and a significant deviation of the frequency of genotypes of both gene markers exist.

\section{References}

1. Jamieson A, Bader S. A guide to forensic DNA profiling. Hoboken: Wiley, 2016. Walsh SJ. Chapter 3, DNA; p. 29-36.

2. Promega Corporation 8/09. Technical manual PowerPlex ESX 16 System. Madison, Wisconsin, USA.

3. Fan H, Chu JY. A brief review of short tandem repeat mutation. Genomics Proteomics Bioinformatics 2007 Feb;5(1):7-14.

4. Marjanovic D, Pojskic N, Davoren J, Kovacevic L, Durmic A, Bakal N, et al. Population data at two short tandem repeat loci D2S1338 and D19S433 in the Sample of multinational Bosnia and Herzegovina residents. J Forensic Sci 2006 Sep; 51(5):1219-20.

5. Pilav A, Pojskić N, Ahatović A, Džehverović M, Čakar J, Marjanović D. Allele frequencies of 15 STR loci in Bosnian and Herzegovinian population. Croat Med J 2017 Jun 14;58(3):250-6.
To conclude the actual difference or similarity in the diversity of autosomal gene markers between these two populations, it is necessary to increase both populations' samples and ensure the correct ethnic and gender representation. In other words, more representative samples are needed. It is also necessary to profile all samples with the same DNA amplification commercial kit to obtain as many genetic markers as possible for comparison.

\section{Acknowledgements}

The Institute of Forensics in Novi Sad kindly provided its DNA profiles for the analysed STR markers, thus enabling a comparison of the same with our data set. The financing of the project was provided by the Institute for Forensic Medicine of the Republic of Srpska.

\section{Conflict of interest}

None.

6. Konjhodžić R, Kubat M, \& Škavić J. Bosnian population data for the 15 STR loci in the Power Plex 16 kit. Int J Legal Med 2004;118(2):119-21.

7. Veselinović I, Kubat M, Furac I, Skavić J, Martinović Klarić I, Tasić M. Allele frequencies of the $15 \mathrm{AmpF}$ ISTR Identifiler loci in the population of Vojvodina Province, Serbia and Montenegro. Int J Legal Med 2004 Jun;118(3):184-6.

8. Jakovski Z, Nikolova K, Furac I, Masic M, Janeska B, Kubat $\mathrm{M}$. Allele frequencies for 15 STR loci in a population from the Republic of Macedonia. Int J Legal Med 2006 Jan;120(1):53-5.

9. Kubat M, Skavić J, Behluli I, Nuraj B, Bekteshi T, Behluli $\mathrm{M}$, et al. Population genetics of the $15 \mathrm{AmpF}$ ISTR Identifiler loci in Kosovo Albanians. Int J Legal Med. 2004 Apr;118(2):115-8. 